\title{
Electrocardiographic Passing Phenomenon (Flying Phenomenon or Yasser's Phenomenon) Conveys the Traditional Cardiovascular Management; Interpretations and Reassurance; Retrospective Observational Study
}

\author{
Yasser Mohammed Hassanain Elsayed*
}

Egyptian Ministry of Health (MOH), Egypt

*Corresponding author: Yasser Mohammed Hassanain Elsayed, Critical Care Unit, Fraskour Central Hospital, Egyptian Ministry of Health (MOH), Egypt.

\begin{abstract}
Background: Dynamic changes during an electrocardiographic recording are common alterations. These changes were commonly reported initially as an abnormal diagnosis by cardiologists despite they are unusual taking the opposite normal side. These changes may be passing as physiological or even transient changes. An electrocardiographic dynamic change with their urgent conversion from abnormal to normal way without any medical interference is the key to the new phenomenon.

Method of study and patients: My case study was an observational retrospective eighteen-case report series. The study was conducted in the Emergency Department and Intensive Care Unit of Fraskour Central Hospital, Dar-Esheefa outpatient clinics, and physician outpatient clinic. The study was taken over nearly 30-months, started from February 1, 2016, and, ended on July 16, 2019.

Results: The age averages for the "Passing phenomenon" were: (Range;17-85, mean; 54.83, median; 56, and mode; 56) with equal sex in the study. The following cases were included in the study: Accelerated junctional rhythm (22.22\%), mimics ST-segment elevation myocardial infarction with accelerated junctional rhythm (5.56\% \%), sinus arrhythmia (22.22\%), bigeminy (11.11\%), trigeminy (11.11\%), quadrigeminy (16.67\% \%), and pentageminy (11.11\%). The averages time elapsed between both ECG tracings (minutes) (Range;1-4, mean; 1.638, median; 1.25, and mode; 1). Reassurance for all cases of the study (100\%). Reassurance with the presence of organic cardiac cause was only in seven cases of this study (38.89\%). Conclusions: The new "Passing phenomenon" is a transient electrocardiographic change that spontaneously reversed within a few seconds to a few minutes without any medical interventions and apparent hemodynamic impact. Reassurance is immediate therapy. The electrophysiological study is the future advised investigation.
\end{abstract}

Keywords: Electrocardiogram; Passing phenomenon; Flying phenomenon; Yasser's phenomenon; Conveys the traditional cardiovascular management; A new cardiovascular phenomenon

Abbreviations: AF: Atrial Fibrillation; AJR: Accelerated Junctional Rhythm; ASD: Atrial Septal Defect; AVN: Atrioventricular Node; BP: Blood Pressure CHB: Complete Heart Block; CTPA: Computed Tomography Pulmonary Angiogram; CXR: Chest X-Rayecg Electrocardiogram; HCV: Hepatitis C Virus; HNJR: High Nodal Junctional Rhythm; HTN: Hypertension; ICU: Intensive Care Unit; IHD: Ischemic Heart Disease; JR: Junctional Rhythms; LNJR: Low Nodal Junctional Rhythm; MI: Myocardial Infarction; NSR: Normal Sinus Rhythm PVC: Premature Ventricular Contraction; RBBB: Right Bundle Branch Block; SA: Sinus Arrhythmia; SAN: Sinoatrial Node; SHD: Structural Heart Disease; SND: Sinus Node Dysfunction; RR: Respiratory Rate; SSS: Sick Sinus Syndrome; STEMI: ST-Elevation Myocardial Infarction; SV: Stroke Volume; VR: Ventricular Rate 


\section{Background}

\section{Junctional rhythm}

Terminology, classifications, and causes: The implicated nomenclature [1] to recognize the type of junctional rhythms (JR) is based on their rate. They are classified as follows:

1) Junctional bradycardia: Ventricular rate $<40 \mathrm{bpm}$.

2) Junction escape rhythm: Ventricular rate 40-60 bpm.

3) Accelerated junctional rhythm (AJR): Ventricular rate of 60-100 bpm.

Table 1: Medications and conditions implicated in junctional rhythm [1,11,25,26].

\begin{tabular}{|c|c|}
\hline Category & Causes \\
\hline \multirow{14}{*}{ - Drug-induced } & Amitriptyline \\
\hline & Antiarrhythmic Class I to IV \\
\hline & - Adenosine \\
\hline & - Beta-blockers \\
\hline & - Calcium channel blockers \\
\hline & Digoxin \\
\hline & Clonidine \\
\hline & Reserpine \\
\hline & Cimetidine \\
\hline & Lithium \\
\hline & Ivabradine \\
\hline & Isoproterenol infusion \\
\hline & Inhalation anesthetics \\
\hline & Narcotics \\
\hline \multirow{10}{*}{ - Cardiovascular } & Sick sinus syndrome \\
\hline & Myocarditis \\
\hline & Pericarditis \\
\hline & Acute and chronic coronary artery disease \\
\hline & - Unstable angina pectoris \\
\hline & - Acute myocardial infarction \\
\hline & Repair of congenital heart disease \\
\hline & Atrial septal defect \\
\hline & Tetralogy of Fallot \\
\hline & Persistent left superior vena cava \\
\hline \multirow{2}{*}{ - Rheumatic } & Acute rheumatic fever \\
\hline & Collagen vascular disease \\
\hline \multirow{3}{*}{ • Neurological } & Neuromuscular disorder \\
\hline & X-linked muscular dystrophy \\
\hline & Intracranial hypertension \\
\hline - Psychiatric & Anorexia nervosa \\
\hline \multirow{3}{*}{ - Autonomic } & Vasovagal simulation (endotracheal suctioning) \\
\hline & Carotid sinus hypersensitivity \\
\hline & Inherited channelopathy \\
\hline \multirow{3}{*}{ - Metabolic } & Hypoxia \\
\hline & Amyloidosis \\
\hline & Sleep apnea \\
\hline - Electrolyte disorders & Hyperkalemia \\
\hline - Endocrinal & Hypothyroidism \\
\hline - Infectious & Lyme disease \\
\hline - Familial & Familial disorder \\
\hline - Traumatic & Chest trauma \\
\hline - Abuse substance & Cannabinoids \\
\hline - Radiological & Radiation therapy \\
\hline
\end{tabular}


4) Junctional tachycardia: Ventricular rate $>100 \mathrm{bpm}$ [1]. If there is blockage for the sinoatrial node (SAN) electrical activity is blocked or is less than the automaticity of the atrioventricular node $(A V N) / H i s$ Bundle a JR starts. Numerous conditions and medications can lead to a diseased SAN and lead to the AVN/His Bundle to take over due to the higher automaticity of the ectopic pacemaker [2-4]. Some of these conditions and medications are listed below [1](Table 1).

Epidemiology: Junctional rhythm is typical among individuals who have a sinus node dysfunction (SND); and 1/600 cardiac patients more than the age of 65 in the US has SND. Patients with sick sinus syndrome (SSS); young children; and athletes who have increased vagal tone may also intermittently exhibit JR; especially during sleep. JRs are equally among males and females [5].

Histopathology: The human heart has several pacemakers to locations in its conduction system which are independently able to conserve the heart beating. However, the rate of a heartbeat is linked the pacemaker site; and as we go down its conduction system; the rate of spontaneous depolarization at pacemaker sites decrease [6-8]. The SAN that can share in the AVN/His Bundle taking over as a pacemaker secondary to their intrinsic automaticity includes SAN fibrosis; atrophy; hypoplasia or amyloidosis. A normal SAN histopathology does not exclude reduced SAN automaticity contributing to a JR $[9,10]$. Pacemaker sites and their depolarization rates are as follow [1]:
1) SAN: $60-100 \mathrm{bpm}$
2) Atria: $<60 \mathrm{bpm}$
3) AVN: $40-60 \mathrm{bpm}$.

4) Ventricles: 20 - 40 beats per minute

Junctional rhythm appears when there is (a) high vagal tone; (b) slowing of the primary atrial pacemaker; usually the SAN; (c) acceleration of a subsidiary pacemaker; (d) atrioventricular (AV) or SA block; or (e) a combination of causes (b) and (d) [11]. The increased automaticity of AV nodal cells is induced by sympathetic overactivity [11]. The accelerated junctional rhythm usually due to an acceleration of AVN pacemaker cells [11]. There was a report; however, of a case in which high nodal junctional rhythm (HNJR) suddenly changed to low nodal junctional rhythm (LNJR) during general anesthesia [11]. HNJR is what appears when the impulse reaches the atrium before the ventricle (P-wave preceding the QRS-complex) [12]. Therefore, the atrial kick is maintained; although it may not be ideally coordinated with ventricular contraction; and the patient's blood pressure (BP) usually remains stable [11].

Presentations: Patients presented with JR have a wide spectrum of symptoms. They may be asymptomatic. Symptoms often based on the causative agent of the JR, for example, a patient presenting with heart failure exacerbation may present with dyspnea, wheezing, and lower extremity edema1. Non-specific physical exam findings include pulsating veins and regular heart rate with the heart rate varying from 20 to over 100 beats per minute [13-15]. The key for a patient who presents with signs and symptoms of a JR is history and physical examination [13-15].

ECG with inverted P-waves in inferior leads (II, III, and aVF) indicates that the atrial activation is spreading from below upwards. It is suggestive of a focus either in the low atrium or high junction. A mid-JR will have no visible $\mathrm{P}$-waves as the $\mathrm{P}$-wave will be within the QRS due to the simultaneous activation of the atria and ventricles. In LNJR the P wave occurs after the QRS, in the ST-segment and is inverted in inferior leads (II, III, and aVF). In left atrial rhythm originating from the lower part, the P-waves are inverted in inferior leads as well as lateral leads [16]. Notching of the QRS-complex in the inferior leads which suggest the crochetage sign in the atrial septal defect (ASD) [16]. Low atrial rhythm can occur with sinus venosus ASD as the sinus node may be defective so that alternate focus arising in the low atrium gives the dominant rhythm [16]. The PR-interval is also shorter in LNJR and low atrial rhythm, more in the former than in the latter, due to obvious reasons [16]. An interesting case report in which left atrial rhythm mimicked myocardial infarction (MI) has been reported recently16. If there is a negative P-wave axis. So, there is now either low atrial rhythm or HNJR (both will have negative P-waves that are seen before the QRS-complex is LNJR with result in P-wave in, or after, the QRS [17].

Differential diagnosis: The following issues implicated in differential diagnosis JR1. Digoxin toxicity, atrioventricular nodal reentrant tachycardia (AVNRT), atrioventricular reentrant tachycardia (AVRT), SAD, high-grade second-degree heart block, and complete heart block (CHB).

Treatment: Junctional rhythm is a benign arrhythmia that often does not demand treatment $[11,12,18,19]$. Treatment for the JR is superfluous [11]. Treatment of a JR primarily based on the cause of the rhythm. If a patient is resistant to medical treatments without passing into junctional tachycardia, IV phenytoin can be given with monitoring for possible hypotension. In the pediatric population, persistent symptomatic junctional tachycardia is an indication for percutaneous radiofrequency ablation. In patients with sick sinus syndrome, or complete or high-grade AV block, a permanent pacemaker is indicated [20-24].

Prognosis: Prognosis is excellent if the JR is urgently recognized by a healthcare provider [1]. Periods of junctional rhythm are not necessarily accompanied by an increase in the mortality rate. If there is a clear cause, such as CHB or SSS, then the morbidity or mortality is directly linked to that and not to the JR mechanism, which is serving as a "backup rhythm" during the periods of bradycardia [26]. Accelerated JR may be a sign of digitalis toxicity. A predominant junctional rhythm may be associated with structural heart disease (SHD), SSS, or both, during which the junctional escape rhythm supersedes the sinus rate and provides a safety mechanism [26]. 


\section{Bigeminy, trigeminy, quadrigeminy, and pentageminy}

Definitions, description, and terminology: Premature ventricular contractions (PVCs) are a type of arrhythmia characterized by premature heart contractions originating in one of the ventricles [27]. PVCs are characterized by premature and bizarre-shaped QRS-complexes that are unusually long (typically $>120 \mathrm{msec}$ ) and appear wide on the ECG. The QRS-complexes are not preceded by a P-wave, and the T-wave is usually large and oriented in a direction opposite the major deflection of the QRS [28].

Depending on whether there are one, two, or three normal beats between each PVC, the rhythm is called bigeminy, trigeminy, or quadrigeminy, etc. $[29,30]$. The two beats are figuratively similar to two twins (hence Bi- + Gemini) indicates the number (2) [30,31]. Tri-is a multiplier suffix indicating the number (3) [31]. Quadri-is a multiplier suffix indicating the number (4) [30,31]. Penta- is a multiplier suffix indicates the number (5) [31]. So, PVCs Bigeminy means a cardiac arrhythmia in which every second beat is a ventricular premature complex (extrasystole) or one sinus beats between extrasystoles [31,32]. Trigeminy means a cardiac arrhythmia in which every third beat is a ventricular premature complex (extrasystole) or two sinus beats between extrasystoles. Quadrigeminy means a cardiac arrhythmia in which every fourth beat is a ventricular premature complex (extrasystole) or three sinus beats between extrasystoles [30,33]. Pentageminy means a cardiac arrhythmia in which every fifth beat is a ventricular premature complex (extrasystole) or four sinus beats between extrasystoles [30].

Epidemiology: It is more prevalent with increasing age [34]. The estimated prevalence of PVCs on standard 12-lead electrocardiography (ECG) was $>1$ PVCs /min occurs in $1 \%$ to $4 \%$ of adults in the general population and $40 \%$ to $75 \%$ in $75 \%$ of healthy persons using on 24- to 48-hour Holter monitoring [35-40]. The prevalence of PVCs depends on the co-morbidities of the patients being screened and the duration of monitoring [41].

Pathophysiology: Traditionally, they have been thought to be relatively benign in the absence of structural heart disease, but they represent an increased risk of sudden death in structural heart disease36. In most cases, premature ventricular contractions have a focal origin [42]. Suggested pathophysiological mechanisms for PVCs are reentry, triggered activity, and enhanced automaticity [28].

Riske factors: Factors that increase the risk of premature ventricular contractions include male sex, advanced age, African-American race, hypertension underlying ischemic heart disease, bundle-branch block, hypomagnesemia, and hypokalemia. The followings causes implicate in PVCs: Hypokalemia, hypomagnesemia, acute myocardial infarction, ischemic heart disease, myocarditis, cardiomyopathy, myocardial contusion, mitral valve prolapse, hypoxia and/or hypercapnia, digoxin, sympathomimetics, tricyclic antidepressants, aminophylline, caffeine, cocaine, amphetamines, alcohol, tobacco, and hypercalcemia $[28,43]$.
Clinical significance, prognosis, and mortality: Patients are usually asymptomatic [28]. The clinical significance of PVCs depends on their frequency, complexity, and hemodynamic response [28]. Ischemic heart disease may be manifested and presented with ventricular arrhythmias like-premature ventricular contractions whatever the type [32]. The vast majority of patients with premature ventricular complexes are considered benign when they occur in patients without apparent SHD [44,45]. PVCs in patients without apparent SHD are safe once we rule out risk factors [44].

Workup: Required workup for premature ventricular contraction includes potassium levels, magnesium level, electrocardiography, and echocardiography [28]. The optimal indications for therapy for premature ventricular contractions have not yet been elucidated [28].

Management and Indications for Therapy: The most common indication for treating PVCs in the absence of structural heart disease (SHD) is the presence of symptoms that are not improved by reassurance $[45,46]$. The presence of symptoms in patients with structural heart diseases is considering primary indication. Elimination of high burden PVCs (10\%) in patients with impaired LV function can be associated with significant improvement of LV function $[47,48]$, even when significant scarring is present $[49,50]$.

\section{Sinus arrhythmia}

Introduction, definition, and description: Normal sinus rhythm (NSR) is the rhythm that arises from the SAN and identifies the known rhythm of the healthy heart [51]. The rate in NSR is generally regular but will vary depending on autonomic inputs into the sinus node. When there is an irregularity in the sinus rate, it is termed sinus arrhythmia (SA) [51]. Sinus arrhythmia is a common variation in the NSR $[52,53]$. Sinus rhythm with a beatto-beat variation in the P-P interval (the time between successive $P$ waves), producing an irregular ventricular rate [54]. Sinus arrhythmia characteristically presents with an irregular rate in which the variation in the R-R interval greater than 0.12 seconds [52] or the P-P-interval of more than $120 \mathrm{~ms}$ ( 3 small boxes) [5355]. There should be a $10 \%$ difference between the maximum and minimum cardiac cycle length [56]. The P-P interval gradually lengthens and shortens cyclically, usually corresponding to the phases of the respiratory cycle [55] with normal sinus P-waves with a constant morphology $[53,54]$ (i.e. no evidence of premature atrial contractions) and constant P-R interval $[53,54]$ (i.e. no evidence of Mobitz I AV block) [54]. So, P-waves are typically mono-form and in a pattern consistent with atrial activation originating from the SAN [52].

Epidemiology and risk factors: Sinus arrhythmia are a normal physiological phenomenon, most commonly seen in young, healthy people [54]. It is seen more often in children and young adults [52]. Sinus arrhythmia is a common incidental sign during routine ECG [52] Sinus arrhythmia is a common sign during telemetry [52,55]. It decreases in prevalence in the elderly, and those with multiple co-morbid conditions include diabetes and heart failure [52]. It is 
considered to be a normal variation found in healthy young adults $[52,55]$. Investigators found that the prevalence of respiratory SA in older individuals was less than 20 percent of that in those less than 31 years of age. Some suspect that this is due to age-related changes in the compliance of arterial walls and diminished vagal responses [57]. Sinus arrhythmia is especially easy to notice with slowing of the heart rate during sleep [56].

Presentation and ECG: Often an asymptomatic and normal finding, the evaluation of sinus arrhythmia is limited. Generally, $\mathrm{SA}$ is at most mildly symptomatic (e.g., palpitations) [55]. On the ECG, sinus rhythm appears as a beat-to-beat variation in the P-P interval. Typically, this variation is greater than $120 \mathrm{msec}$ : the P-P interval increases and decreases with inspiration and exhalation [52-55]. The P-wave morphology usually does not change56. If it does change, the changes are phasic and the $\mathrm{P}$ waves do not appear retrograde [56]. The heart rate varies due to reflex changes in vagal tone during the different stages of the respiratory cycle. Inspiration increases the heart rate by decreasing vagal tone. With the onset of expiration, vagal tone is restored, leading to a subsequent decrease in heart rate [54].

Pathogenesis and its variants: Respirations lead to vagal stimuli resulting in $\mathrm{R}-\mathrm{R}$ interval variations [52]. During respiration, the intermittent vagus nerve activation occurs, which results in beat to beat variations in the resting heart rate [52]. Atrioventricular conduction is normal [56]. Presumably, it may due to age-related decreases in carotid distensibility and baroreceptor reflex sensitivity [54]. Investigation on the pathogenesis of sinus arrhythmia continues but three main theorized mechanisms exist at this time. These include respiratory-phasic, non-respiratory-nonphasic, and non-respiratory-ventriculo-phasic sinus arrhythmia [52]. Respiratory SA is a common sign. It is a normal alteration in cardiac rhythm generated from the stimulation of the vagus nerve and changes in cardiac filling pressures during respiration [52]. Some studies have begun to associate sinus rhythm with obesity, diabetes mellitus, and hypertension (HTN) while others continue to support a reduction in sinus arrhythmia when these conditions are present [52]. One study suggested that the mediation of SA occurs through vagal stimulation $[53,58]$. The study suggested that baroreceptor signaling plays an important role in the generation of respiratory SA [59]. The investigators have evaluated the effects of diabetes mellitus on SA. They found that in patients with diabetes there is a reduction in SA, which investigators attributed to the autonomic effects of the disease [60]. In non-respiratory SA, electrocardiograms will appear similar to the respiratory type. The two differ in that nonrespiratory SA is not associated with the respiratory cycle. While it can occur within healthy individuals, more often this type correlates with an underlying pathology [61]. This type was reported with digitalis overdose and traumatic intracerebral hemorrhage that associated with vasospasm, hypoxia, and increased intracranial pressure [62]. The ventriculo-phasic SA, when present, typically occurs in patients with third-degree $\mathrm{AV}$ block [52,53]. It occurs if there is coexistence for both sinus rhythm and high-grade or complete AV block. So, it is characterized by shorter P-P intervals when they enclose QRS-complexes and longer P-P-intervals when no QRS-complexes are enclosed [53]. However, they show good correlations with PVCs. Currently, the suggested mechanisms that ventriculophasic SA results due to a prolonged cardiac filling resulting in increased stroke volume (SV). The increased SV triggers a carotid baroreceptor response. The ECG represents an interval shortening between the QRS and subsequent P-wave [52].

Differential diagnosis: A differential diagnosis for sinus arrhythmia would include frequent premature atrial contractions, $\mathrm{AF}$, second-degree AV block, Mobitz I (Wenckebach phenomenon), type I sinoatrial exit block, and CHB [51,54]. Care should be taken to rule out other causes of arrhythmia including atrial fibrillation, flutter or multifocal atrial tachycardia with an ECG. P-waves will show uniform morphology [52].

Treatment: Upon confirming the diagnosis of SA on ECG, there are no further recommendations regarding treatment $[52,55]$. The patients rarely need cardiological evaluation for additional any treatment [52].

Prognosis: It is most common in young, healthy patients. Typically, its presence is a prognostic indicator for good cardiovascular health $[52,53]$. Loss of SA may indicate underlying heart failure or structural heart disease [52].

\section{Case Presentations}

The author had reported eighteen-cases of variants diagnosis through nearly 30-months, started from January 13, 2016, and, ended on Jul 6, 2019. The study was conducted in the Emergency Department and Intensive Care Unit of Fraskour Central Hospital, Dar Eshefa outpatient clinics, and physician outpatient clinic. The study was taken over nearly 30-months, started from January 13, 2016, and, ended on July 16, 2019. The eighteen reported cases were variable in diagnosis. ECG and clinical status are the main judges for the study. The ECG changes were continued to show dynamic ST-segment, T-wave, and arrhythmic changes. Two spontaneous ECG tracings were taken. The repeat ECG tracing was performed a few seconds to a few minutes. A repeat ECG tracing were mostly demonstrated normal sinus rhythm or even abnormal basic rhythm. Resolved transient ST-elevation myocardial infarction (STEMI) with negative biomarkers also present. The later ECG manifestations may mimic acute myocardial injury. All these ECG changes mostly not related to the relevant basic diagnosis of study diseases. These changes were transient. The changes were completely reversed without any medical or procedural intervention. The reversibility had happened within a few seconds to a few minutes. Myocardial necrosis was excluded using the negativity of cardiac enzymes (troponins) and typical ECG criteria for the evolution of acute myocardial infarction. There was no history of cardiac disease in her past for most cases. Most cases were undergone a transthoracic echocardiogram (TTE) and troponin test. There was 
a different diagnosis for all the study cases. There are four cases of accelerated junctional rhythm, one case of ST-segment elevation with accelerated junctional rhythm, four cases of sinus arrhythmia, two cases of bigeminy, two cases of trigeminy, three cases of quaderigeminy, and two cases of pentageminy. For more details for the study cases, see (Table 2).

Table 2: Summary of the history, clinical, and management data for the study cases.

\begin{tabular}{|c|c|c|c|c|c|c|c|c|c|c|c|c|}
\hline $\begin{array}{c}\text { Case } \\
\text { No. }\end{array}$ & Age & Sex & Occupation & $\begin{array}{l}\text { The main } \\
\text { complaint }\end{array}$ & $\begin{array}{c}\text { BP } \\
\mathrm{mg} \mathrm{Hg}\end{array}$ & Pulse & $\mathbf{R R}$ & $\begin{array}{c}02 \\
\text { Sat. } \\
\%\end{array}$ & $\begin{array}{l}\text { Time between } \\
\text { both ECGs }\end{array}$ & $\begin{array}{l}\text { Basic di- } \\
\text { agnosis }\end{array}$ & Cardiac diagnosis & $\begin{array}{l}\text { Reassurance } \\
\text { But with organic } \\
\text { cause }\end{array}$ \\
\hline 1 & 17 & M & Student & Chest pain & $130 / 50$ & 80 & 15 & 97 & 1 & Cold & Mimic $\mathrm{MI}+\mathrm{AJR}^{*}$ & - \\
\hline 2 & 56 & M & $\begin{array}{l}\text { Teacher } \\
\text { Housewife }\end{array}$ & Chest pain & $130 / 80$ & 74 & 16 & 98 & 2 & $\begin{array}{l}\text { C. disc } \\
\text { prolapse }\end{array}$ & AJR+ Sinus arrhy & - \\
\hline 3 & 43 & $\mathrm{~F}$ & Farmer & Chest pain & $110 / 60$ & 90 & 14 & 99 & 2 & Myositis & AJR & - \\
\hline 4 & 47 & $\mathrm{M}$ & Housewife & Chest pain & $130 / 80$ & 70 & 18 & 96 & 1 & Cold & AJR & - \\
\hline 5 & 56 & $\mathrm{~F}$ & Worker & Follow up & $140 / 90$ & 76 & 12 & 95 & 2 & Follow up & AJR & + (Myoperic) \\
\hline 6 & 28 & M & Carpenter & Follow up & $130 / 80$ & 85 & 16 & 99 & 1 & Anxiety & Quadrigeminy & - \\
\hline 7 & 76 & M & Housewife & Palpitations & $140 / 90$ & 100 & 14 & 96 & 2 & Anxiety & Pentageminy & - \\
\hline 8 & 70 & $\mathrm{~F}$ & Teacher & Headache & $190 / 110$ & 100 & 24 & 95 & 1.5 & $\mathrm{CVA}+\mathrm{HTN}$ & Pentageminy & + \\
\hline 9 & 49 & M & Driver & Palpitations & $120 / 70$ & 64 & 18 & 98 & 1 & Old IMI & Trigeminy & + \\
\hline 10 & 43 & M & Worker & $\begin{array}{l}\text { abdominal } \\
\text { pain }\end{array}$ & $140 / 80$ & 96 & 16 & 99 & 1 & Renal pain & Quadrigeminy & - \\
\hline 11 & 75 & M & Housewife & $\begin{array}{l}\text { abdominal } \\
\text { pain }\end{array}$ & $90 / 60$ & 90 & 22 & 93 & 1 & $\begin{array}{l}\text { Ischemic } \\
\text { colitis }\end{array}$ & Trigeminy & + \\
\hline 12 & 68 & $\mathrm{~F}$ & Housewife & Follow up & $150 / 90$ & 96 & 14 & 97 & 3 & HTN & Quadrigeminy & + \\
\hline 13 & 67 & $\mathrm{~F}$ & Housewife & Dyspnea & $90 / 60$ & 110 & 28 & 93 & 3 & $\mathrm{AF}+$ ? APE & Bigeminy & + \\
\hline 14 & 50 & $\mathrm{~F}$ & Housewife & Palpitations & $100 / 60$ & 120 & 17 & 94 & 2 & $\mathrm{AF}$ & Bigeminy & + \\
\hline 15 & 66 & $\mathrm{~F}$ & Housewife & Follow up & $100 / 80$ & 90 & 15 & 98 & 1 & Follow up & Sinus arrhythmia & - \\
\hline 16 & 57 & $\mathrm{~F}$ & Worker & Preoperative & $120 / 70$ & 76 & 16 & 99 & 1 & $\begin{array}{l}\text { Preopera- } \\
\text { tive }\end{array}$ & Sinus arrhythmia & - \\
\hline 17 & 34 & M & Housewife & Follow up & $110 / 70$ & 66 & 14 & 97 & 4 & Follow up & Sinus arrhythmia & - \\
\hline 18 & 85 & $\mathrm{~F}$ & & Palpitations & $140 / 80$ & 60 & 13 & 95 & 3 & Anxiety & Sinus arrhythmia & - \\
\hline
\end{tabular}

AF: Atrial Fibrillation; APE: Acute Pulmonary Embolism; AJR: Accelerated Junctional Rhythm; Arrhy: arrhythmia; CVA: cerebrovascular accident; HTN: hypertension; IMI: inferior myocardial infarction; MI: myocardial infarction; Myoperic: myopericarditis

\section{Case 1: Mimic acute ST-segment elevation MI with accelerated junctional rhythm in pleurisy and cold}

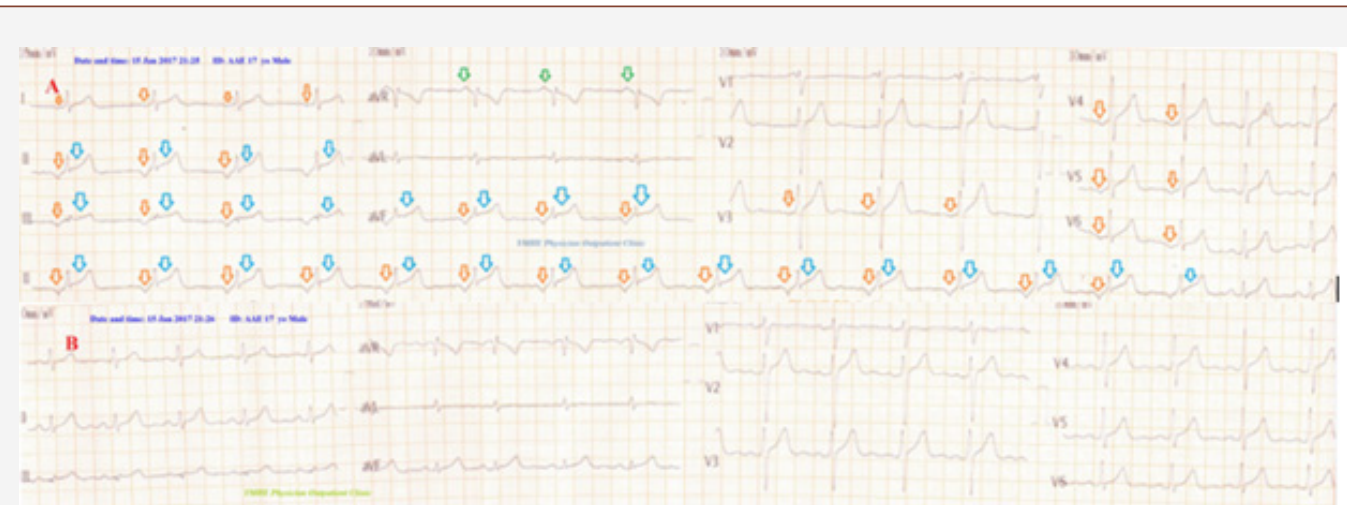

Figure 1: A. the initial ECG tracing showing ST-segment elevation in all inferior ECG leads (II, III, aVF) (blue arrows) with P-wave inversion (orange arrows) through lead I, all inferior ECG leads (II, III, aVF), and V3-6, upright P-wave in aVR (green arrows) with VR;78 bpm.

B. ECG tracing one minute later showing normalization of all above changes.

A 17-year-old single Egyptian male student presented to physician outpatient clinic with pleuritic chest pain. The patient had a recent history for an attack of a common cold. Upon examination BP of $130 / 70 \mathrm{mmHg}$, the pulse of $80 \mathrm{bpm}$, RR of $15 \mathrm{bpm}$, the temperature of $37.4^{\circ} \mathrm{C}$, and $\mathrm{O}_{2}$ saturation with pulse oxymetry $97 \%$. Chest examining was showing pleuritic rub on listening. No more relevant 
clinical data were noted during the clinical examination. The ECG recordings were showing ST-segment elevation in all inferior ECG leads (II, III, aVF) with P-wave inversion through lead I, all inferior ECG leads (II, III, aVF), and V3-6, upright P-wave in aVR with VR;78 bpm (Figure 1A). ECG recordings were taken one minute later and were completely normal without any medications (Figure 1B). No electrolytes profile and thyroid function tests abnormalities were detected. Troponin test was below the normal level. CXR film was showing increased bronchovascular marking. Echocardiography was normal. Pleurisy was managed only with ibuprofen (tab., 400 $\mathrm{mg} / 8$ hours for 5 days). No recurrence for above ECG abnormalities on later serial ECG on follow up.

\section{Case 2: Accelerated junctional rhythm and sinus arrhythmia with cervical disc prolapse}

A A 56-year-old married Egyptian male teacher patient presented to POC with musculoskeletal chest pain. Chest pain associated with bilateral arm pain with weakness, paraesthesia, and numb- ness. The patient gives a history of lumbosacral herniated disc prolapse with the surgical repair 9 years ago. Upon examination BP of $130 / 80 \mathrm{mmHg}$, the pulse of $74 \mathrm{bpm}$, RR of $16 \mathrm{bpm}$, the temperature of $36.6{ }^{\circ} \mathrm{C}$, and $\mathrm{O}_{2}$ saturation with pulse oxymetry $98 \%$. Local tenderness was elicited. No more relevant clinical data were noted during the clinical examination. The ECG recordings were showing accelerated junctional rhythm in the inferior leads; II, III, aVF (inverted P-wave), and aVR (upright P-wave) with sinus arrhythmia (Figure 2A). ECG recordings were taken two minutes later and were completely normal without any medications (Figure 2B). Echocardiography and troponin test were normal. MRI of the cervical spine showing herniated disc prolapse between $\mathrm{C}_{4}$ and $\mathrm{C}_{5}$ vertebrae with spinal stenosis. No recurrence of accelerated junctional rhythm on later serial ECG tracings. Electrolytes profile, thyroid function tests, full blood picture, kidney function tests were done. All results of the required investigations were normal. The patient was referred to the neurosurgeon after reassurance for further evaluation and management.

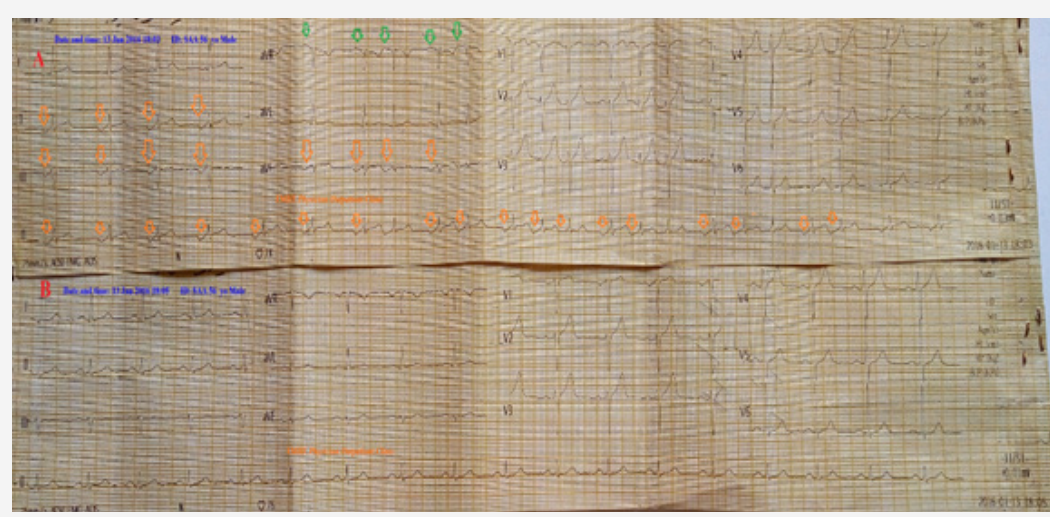

Figure 2: A. the initial ECG tracing showing P-wave inversion (orange arrows) through all inferior ECG leads (II, III, aVF) and upright P-wave in aVR (green arrows) and sinus arrhythmia with VR; 95 bpm.

B. ECG tracing two minutes later showing normalization of all above changes.

\section{Case 3: Accelerated junctional rhythm with myositis}

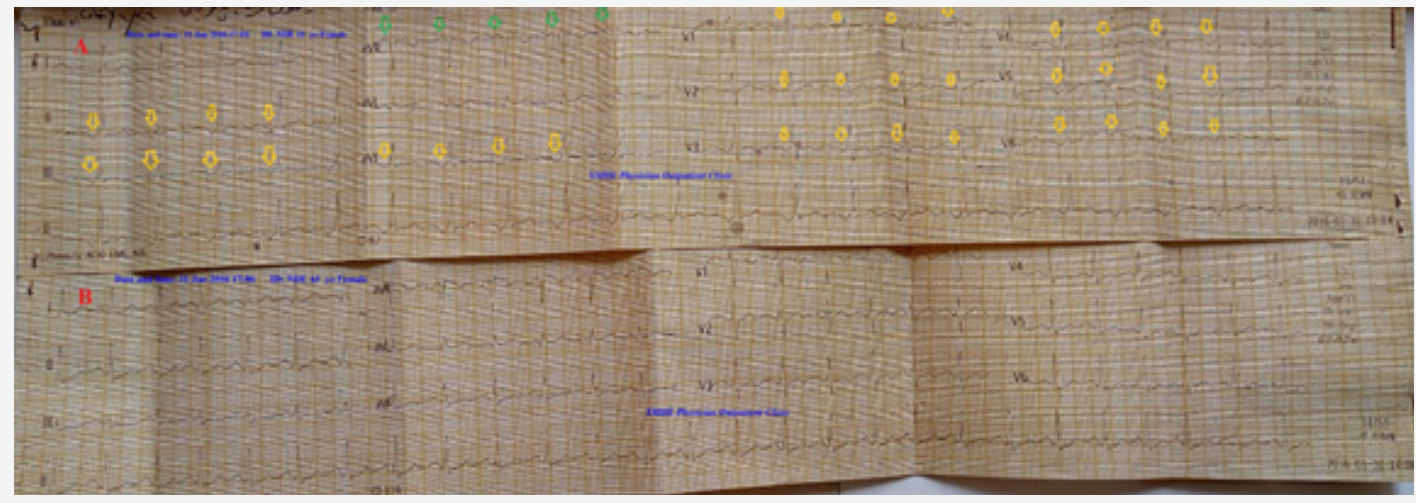

Figure 3 : A. the initial ECG tracing showing P-wave inversion through all inferior ECG leads (II, III, aVF) (gold arrows) and upright P-wave in aVR (green arrows), all anterior leads (V1-6) (gold arrows) with VR; 90 bpm.

B. ECG tracing two minutes later showing normalization of all above changes.

A 43-year-old married housewife Egyptian female patient presented to POC with musculoskeletal chest pain. Upon examina- tion BP of $110 / 60 \mathrm{mmHg}$, the pulse of $90 \mathrm{bpm}$, the temperature of $37.1^{\circ} \mathrm{C}$, RR of $14 \mathrm{bpm}$, and $\mathrm{O}_{2}$ saturation with pulse oxymetry $99 \%$. 
Local tenderness was elicited. The ECG recordings were showing accelerated junctional rhythm in the inferior leads (II, III, aVF) and all anterior leads (V1-6) (Figure 3A). ECG recordings were taken two minutes later and were completely normal without any medications (Figure 3B). Electrolytes profile, thyroid function tests, full blood picture, kidney function tests, and CPK were done. Otherwise of leukocytosis (14400) and increased CPK (473 IU/L), All other results of required investigations were normal. The echocardiography was normal. Troponin test was below the normal level. The patient was managed only with diclofenac potassium (tab., $25 \mathrm{mg} / 8$ hours for 5 days). No recurrence for above ECG abnormalities on later serial ECG on follow up.

\section{Case 4: Accelerated junctional rhythm in pleurisy and cold}

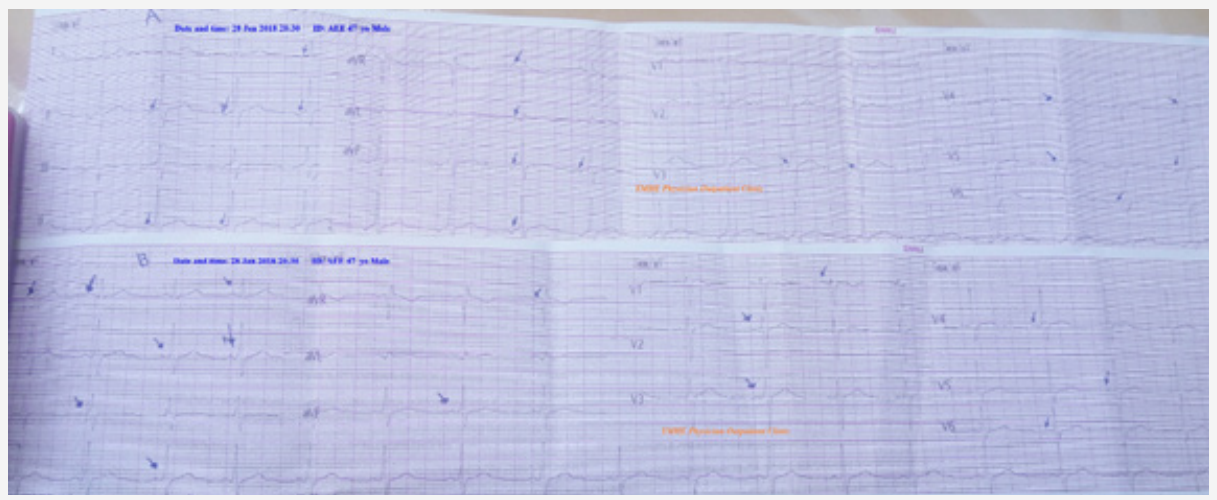

Figure 4 : A. the initial ECG tracing showing P-wave inversion (blue arrows) through all ECG leads aVF) except aVR, V1, and V2(upright P-wave) with VR; $70 \mathrm{bpm}$.

B. ECG tracing one minute later showing normalization of all above changes.

A 47-year-old married male heavy smoker farmer Egyptian patient presented to the POC with pleuritic chest pain. The patient had a recent history of a common cold. Upon examination BP of 130/80 $\mathrm{mmHg}$, the pulse of $70 \mathrm{bpm}, \mathrm{RR} ; 18 \mathrm{bpm}$, the temperature of $37.4{ }^{\circ} \mathrm{C}$, and $\mathrm{O}_{2}$ saturation with pulse oxymetry $96 \%$. Chest examining was showing pleuritic rub on listening. No more relevant clinical data were noted during the clinical examination. The ECG recordings were showing P-wave inversions through all ECG leads except with VR;70 bpm (Figure 4A). ECG recordings were taken one minute later and were completely normal without any medications (Figure 4B). No electrolytes profile and thyroid function tests abnormalities were detected. Troponin test was below the normal level. CXR film was showing increased bronchovascular marking. Echocardiography was normal. Pleurisy was managed only with diclofenac (tab., $25 \mathrm{mg} / 8$ hours for 5 days). No recurrence for above ECG abnormalities on later serial ECG tracings follows up.

\section{Case 5: Accelerated junctional rhythm with suspected chronic myopericarditis}

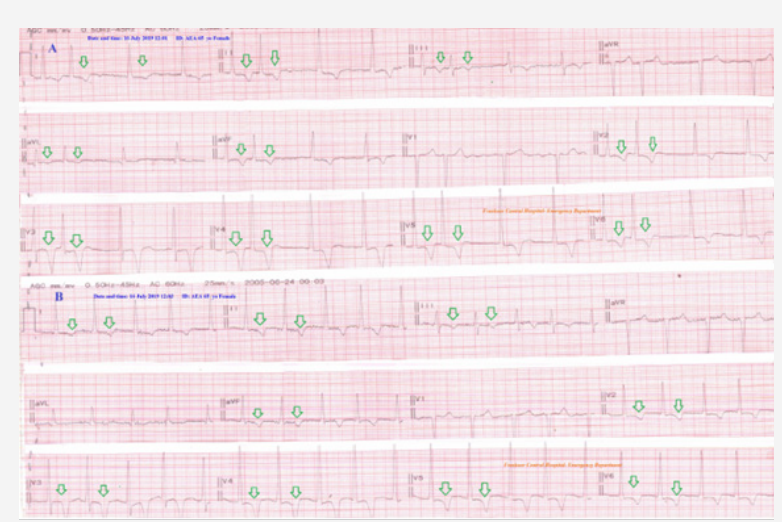

Figure 5 : A. the initial ECG tracing showing absent P-wave inversion through all ECG leads and T-wave inversion in all ECG leads except aVR, aVL, V1 with VR (green arrows) with 76 bpm.

B. ECG tracing two minutes later showing the only normalization of ECG regularity.

A 65-year-old married Egyptian female housewife presented to the emergency room for follow up. The patient gives a history of IHD. Upon examination BP of 140/90 mmHg, the pulse of $76 \mathrm{bpm}$, the temperature of $36.9^{\circ} \mathrm{C}$, RR of $12 \mathrm{bpm}$, and $\mathrm{O}_{2}$ saturation with pulse oxymetry $95 \%$. No more relevant clinical data were noted during the clinical examination. The ECG recordings were showing 
accelerated junctional rhythm (absent P-wave in all leads), sinus arrhythmia, and wide-spread T-wave inversions (Figure 5A). ECG recordings were taken two minutes later and were completely normal without any medications (Figure 5B). The patient admitted in ICU for ECG changes. Echocardiography and troponin test were normal. No recurrence of accelerated junctional rhythm on later serial ECG tracings. Otherwise of leukocytosis (16100) and increased ESR of 1st hour (53 IU/L), electrolytes profile, thyroid function tests, full blood picture, kidney function tests were normal. No recurrence for above ECG abnormalities on later serial ECG tracings follows up. The patient was discharged after reassurance with further follows up.

\section{Case 6: Quadrigeminy with anxiety}

A 28-year-old married Egyptian male worker presented to the hospital outpatient clinic for a regular check-up. The patient had a recent history of anxiety. The patient denied any history of cigarette smoking, drugs, any special diet/habit and any history of cardiac, thyroid, or other relevant diseases, psychiatric problems, and exhaustion. Upon examination, the patient was conscious, alert with $\mathrm{BP}$ of $130 / 70 \mathrm{mmHg}$, the pulse rate of $85 \mathrm{bpm}$, RR of $16 \mathrm{bpm}$, and the temperature of $36.8{ }^{\circ} \mathrm{C}$, and $\mathrm{O}_{2}$ saturation with pulse oxymetry 99\%. No more relevant clinical data were noted during the clinical examination. The ECG recordings were showing quadrigeminy through all ECG leads (Figure 6A). ECG recordings were taken one minute later and were completely normal (Figure 6B). Patient pulse during the first ECG showed irregular regularity while pulse examination was completely regular during the second ECG. Quadrigeminy disappears without any medical interventions. No recurrence of quadrigeminy on later serial ECG tracings. Electrolytes profile, thyroid function tests, full blood picture, kidney function tests, and echocardiography were done. All results of the required investigations were normal. The patient left the hospital after reassurance.

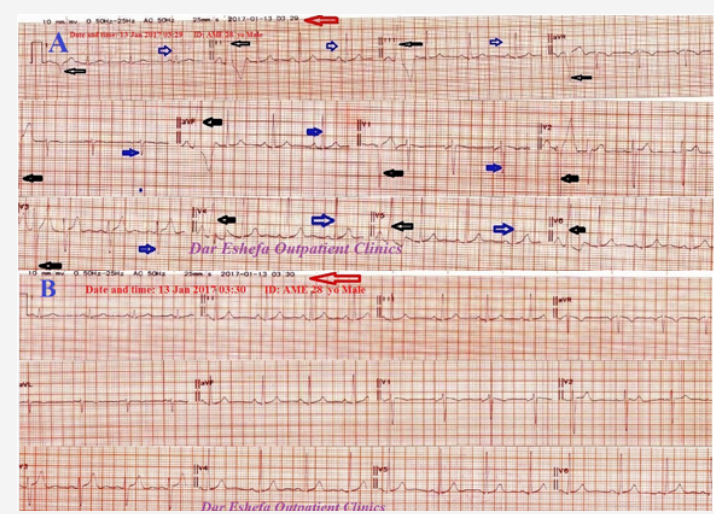

Figure 6 : A. The initial ECG tracing showing quadrigeminy during patient's anxiety from electrocardiographic electricity through all ECG leads (black arrows indicate premature ventricular contraction (quadrigeminy) followed by three normal sinus beats (blue arrows). Red arrow indicates the time and date of ECG.

B. The second ECG tracing (one minute after the first ECG) showing complete showing normalization of all above changes. Red arrow indicates the time and date of ECG.

\section{Case 7: Pentageminy with anxiety}

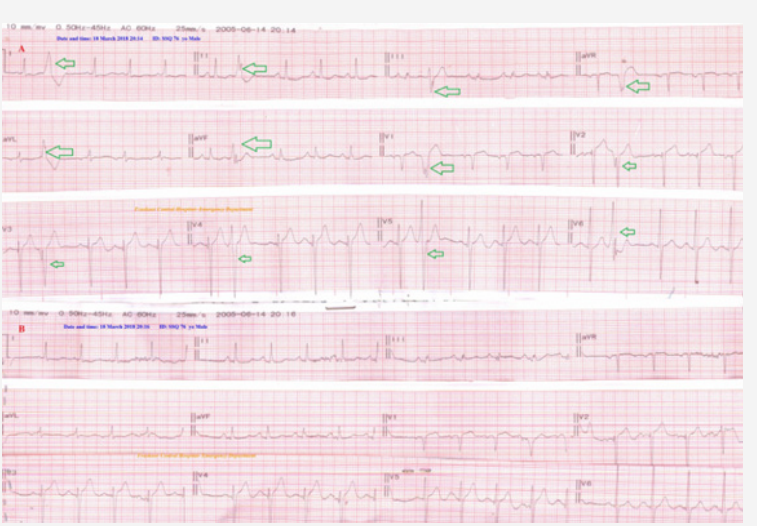

Figure 7 : A. The initial ECG tracing showing pentageminy during patient's anxiety from electrocardiographic electricity through all ECG leads (green arrows indicate premature ventricular contraction (pentageminy) followed by four normal sinus beats.

B. The second ECG tracing (two minutes after the first ECG) showing complete showing normalization of all above changes.

A 76-year-old single Egyptian carpenter male patient presented to the emergency room with palpitations. The patient appeared anxious. Recent history of socio-familial stress. Upon examination BP of $140 / 90 \mathrm{mmHg}$, the pulse of $100 \mathrm{bpm}, \mathrm{RR}$ of $14 \mathrm{bpm}$, the tem- 
perature of $36.3{ }^{\circ} \mathrm{C}$, and $\mathrm{O}_{2}$ saturation with pulse oxymetry $96 \%$ No more relevant clinical data were noted during the clinical examination. The ECG recordings were showing pentageminy through all ECG leads with VR;100 bpm. (Figure 7A). ECG recordings were taken two minutes later and were the disappearance of pentageminy without any medications (Figure 7B). No electrolytes profile or thyroid function tests abnormalities were detected. Echocardiography was normal. No recurrence for above ECG pentageminy on later serial ECG tracings follows up.

\section{Case 8: Pentageminy with cerebrovascular accident}

A 70-year-old single Egyptian housewife female patient presented to the emergency room with a severe headache. The patient had admitted in ICU as a cerebrovascular accident. Upon examina- tion BP of $190 / 110 \mathrm{mmHg}$, the pulse of $100 \mathrm{bpm}$, RR of $24 \mathrm{bpm}$, the temperature of $36.9^{\circ} \mathrm{C}$, and $\mathrm{O}_{2}$ saturation with pulse oxymetry $95 \%$. Neurological revealed right hemiplegia with GCS was [13]. No more relevant clinical data were noted during the clinical examination. Brain CT showed subarachnoid hemorrhage. The ECG recordings were showing pentageminy through all ECG leads, ST-segment depressions in the all anterior leads with sinus tachycardia (VR;100 bpm) (Figure 8A). ECG recordings were taken one and half a minute later and were the disappearance of pentageminy without any medications (Figure 8A). No electrolytes profile or thyroid function tests abnormalities were detected. Chest X-ray (CXR) film was showing cardiomegaly with enlargement of the left ventricle. Echocardiography was left ventricular systolic dysfunction. No recurrence for above ECG pentageminy on later serial ECG tracings follows up.

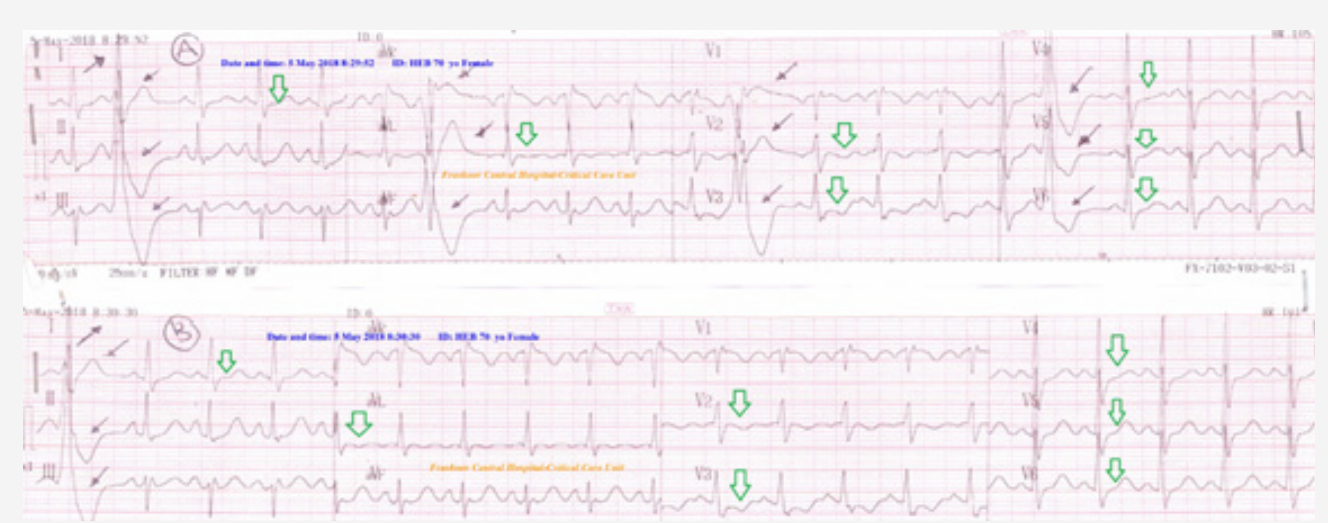

Figure 8 : A. The ECG recordings were showing pentageminy through all ECG leads (black arrows)., ST-segment depressions in the all anterior leads (green arrows) with sinus tachycardia (VR;100 bpm).

B. ECG recordings were taken one and half a minute later and were the disappearance of pentageminy with very few and still ST-segment depressions (green arrows).

\section{Case 9: Trigeminy with anxiety and old IMI}

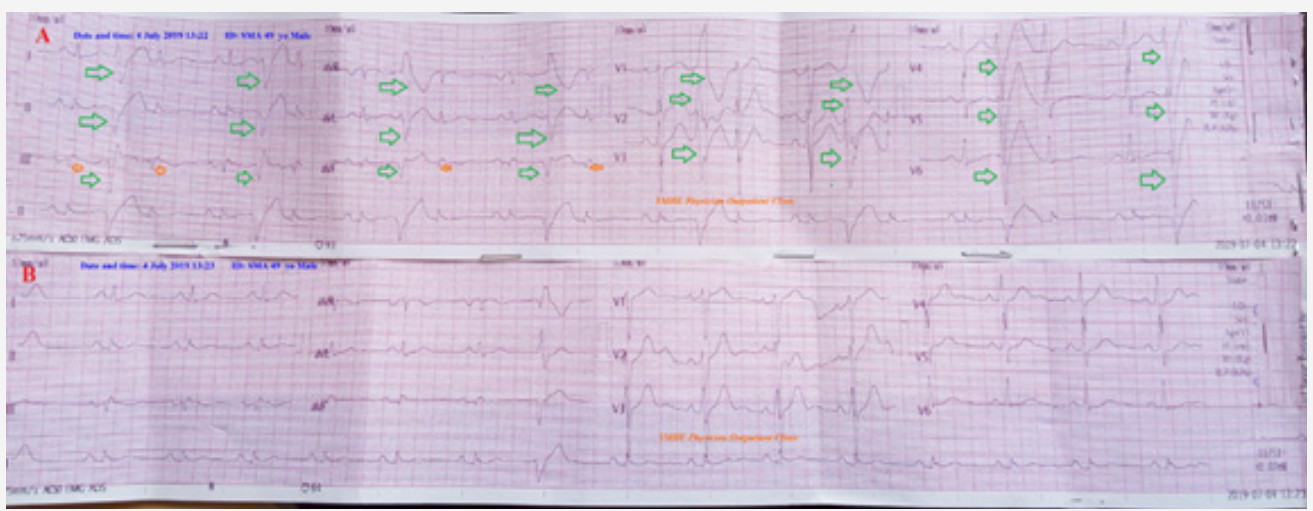

Figure 9 : A. The initial ECG tracing showing trigeminy beats (green arrows) with evidence of inferior myocardial infarction (orange arrows). B. The second ECG tracing (one minute after the first ECG) showing very few PVCs with nearly complete showing normalization of all above changes.

A 49-year-old married Egyptian teacher male patient presented to the POC with palpitations. The patient appeared anxious. Recent history of socio-familial stress. There was a history of old MI. Upon examination BP of $120 / 70 \mathrm{mmHg}$, the pulse of $64 \mathrm{bpm}$; irregular, RR of $1 \mathrm{bpm}$, the temperature of $36.7^{\circ} \mathrm{C}$, and $\mathrm{O}_{2}$ saturation with pulse oxymetry of $98 \%$. No more relevant clinical data were noted during the clinical examination. The ECG recordings were showing trigeminy through all ECG leads with VR;66 bpm (Figure 9A). ECG recordings were taken one minute later and were the disappearance of trigeminy without any medications (Figure 9B). No electro- 
lytes profile or thyroid function tests abnormalities were detected Echocardiography was normal. No recurrence for above ECG trigeminy on later serial ECG tracings follows up.

\section{Case 10: Mixed ventricular and atrial quaderigeminy with left renal pain}

A 43-year-old married driver Egyptian male patient presented to the emergency room with left loin pain. Upon examination BP of $140 / 80 \mathrm{mmHg}$, the pulse of $96 \mathrm{bpm}$, RR of $16 \mathrm{bpm}$, the temperature of $36.9{ }^{\circ} \mathrm{C}$, and $\mathrm{O}_{2}$ saturation with pulse oxymetry $99 \%$. The ECG recordings were showing quaderigeminy (Figure 10A). ECG re- cordings were taken one minute later and were completely normal without any medications (Figure 10B). Electrolytes profile, thyroid function tests, full blood picture, kidney function tests, echocardiography, urine analysis, and abdominal ultrasound were done. Otherwise, present left ureteric stone on abdominal ultrasound showed and heavy oxalate salts (+++) on urine analysis, all other investigations were normal. The patient was managed only with diclofenac potassium $75 \mathrm{mg}$ amp with reassurance for quadergeminy. No recurrence for above ECG abnormalities on later serial ECG tracings follow up. The patient was referred for a urologist for follows up.

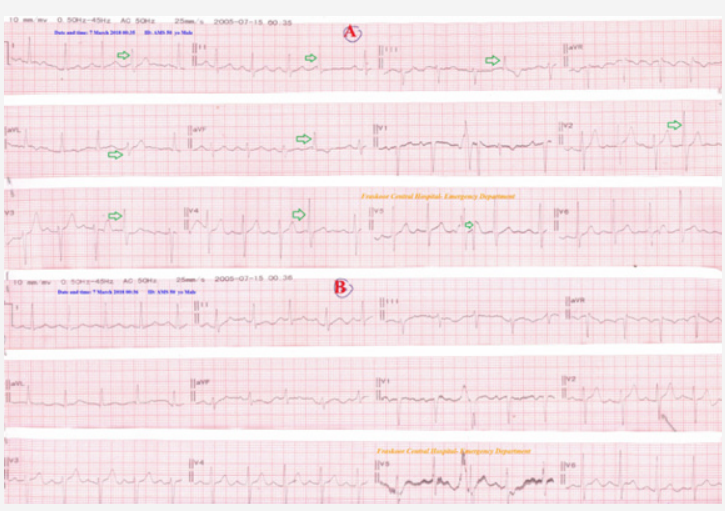

Figure 10 : A. The initial ECG tracing showing mixed ventricular and atrial quaderigeminy through most ECG leads (green arrows indicate premature ventricular contraction (quaderigeminy) followed by three normal sinus beats.

B. The second ECG tracing (one minute after the first ECG) showing complete showing normalization of all above changes.

\section{Case 11: Trigeminy with suspected ischemic colitis}

A 75-year-old married Egyptian male worker patient presented to the emergency room with left abdominal and periumbilical pain. The patient gave a recent history of diarrhea mild rectal bleeding and fever. The patient gave a history of old MI, hypertension, and post-coronary artery bypass grafting. The patient also gave a history of HCV with antiviral treatment 6 years ago. Upon examination BP of $90 / 60 \mathrm{mmHg}$, the pulse of $90 \mathrm{bpm}$, RR of $22 \mathrm{bpm}$, the temperature of $38,1^{\circ} \mathrm{C}$, and $\mathrm{O}_{2}$ saturation with pulse oximetry $93 \%$. Local abdominal tenderness was elicited. The patient was admitted to the internal ward and was managed with IV fluids, antibiotics, and oxygen. The ECG recordings were showing PVCs-trigeminy through all ECG leads (Figure 11A). ECG recordings were taken one minute later and were near completely normal (Figure 11B). The patient was symptomatically improved with BP normalization within 6 hours. Initial laboratory workup was showing leukocytosis (17000), mild anemia (Hb; 9 g/dl. 3\%, RBC; $3.8 \mathrm{M}$ ), elevated serum creatinine (1.7). The patient insisted on hospital discharge despite incomplete workup. ECG recordings were taken on the patient discharge with no trigeminy.

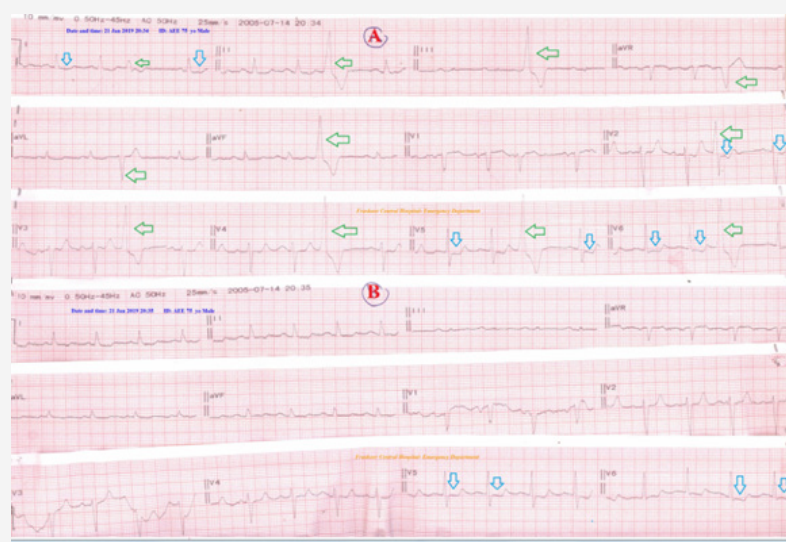

Figure 11 : A. The initial ECG tracing showing trigeminy beats in most ECG leads (green arrows) with evidence of IHD (ST-segment depressions) in I, V2, V5, and V6 leads with VR of 82.

B. The second ECG tracing (one minute after the first ECG) showing complete showing normalization of all trigeminy with still some STsegment depressions in I, V5, and V5 leads. 


\section{Case 12: Quaderigeminy with hypertension}

A 68-year-old married housewife Egyptian female patient presented to the emergency room for follow up. The patient had a history for hypertension on oral captopril tab., (25 mg OD). Upon examination BP of $150 / 90 \mathrm{mmHg}$, the pulse of $96 \mathrm{bpm}$, the temperature of $36.2{ }^{\circ} \mathrm{C}$, RR of $14 \mathrm{bpm}$, and $\mathrm{O}_{2}$ saturation with pulse oxymetry of $97 \%$. The ECG recordings were showing quaderigem- iny in most leads (Figure 12A). ECG recordings were taken three minutes later and were completely normal without any medications (Figure 12B). Electrolytes profile, thyroid function tests, full blood picture, and kidney function tests were done. All results of the required investigations were normal. Echocardiography was normal. The patient was managed only with reassurance for quaderigeminy. No recurrence for above ECG abnormalities on later serial ECG tracings follows up.

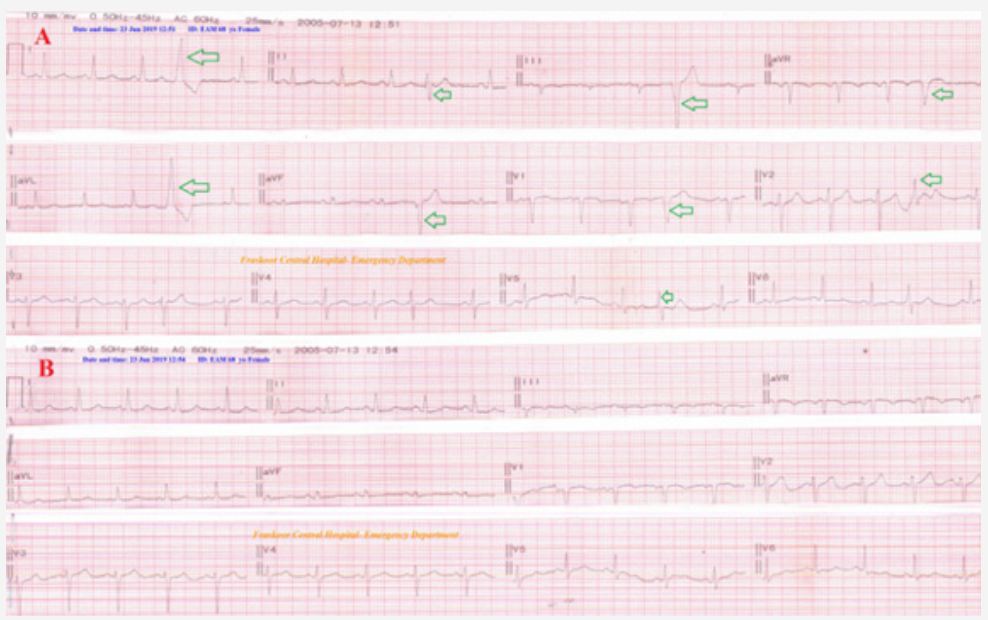

Figure 12 : A. The initial ECG tracing showing quaderigeminy beats in most ECG leads (green arrows).

B. The second ECG tracing (three minutes after the first ECG) showing complete showing normalization of all quaderigeminy.

\section{Case 13: Bigeminy with AF, RBBB, and suspected acute pulmonary embolism}

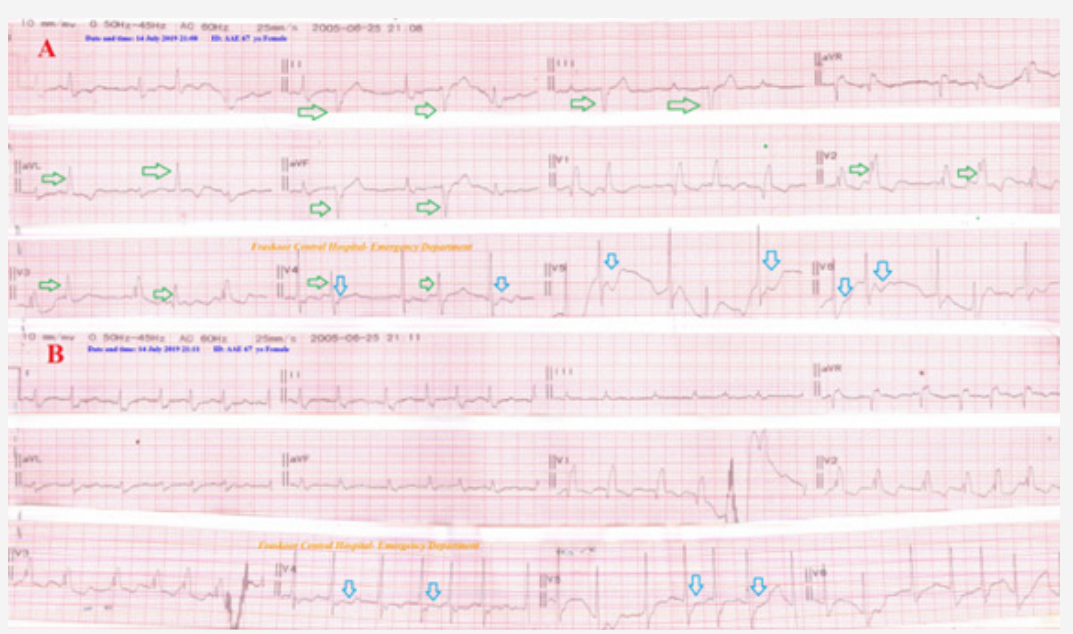

Figure 13 : A. The initial ECG tracing showing AF, RBBB, ST-depressions in V4-6 leads (blue arrows), and bigeminy through most ECG leads (green arrows).

B. The second ECG tracing (three minutes after the first ECG) showing completely disappearance only for bigeminy.

A 67-year-old married Egyptian female housewife presented to the emergency room with dyspnea and palpitations. The patient gave a history of IHD. Upon examination, the patient appeared dyspneic, tachypneic, alert with the blood pressure of $90 / 60 \mathrm{mmHg}$, the pulse rate of $110 \mathrm{bpm}, \mathrm{RR}$ of $28 \mathrm{bpm}$, the temperature of $37.5^{\circ} \mathrm{C}$, and $\mathrm{O}_{2}$ saturation with pulse oxymetry of $93 \%$. No more relevant clinical data were noted during the clinical examination. The ECG recordings were showing AF, RBBB, ST-depressions in V4-6 leads, and bigeminy through all ECG leads (Figure 13A). ECG recordings were taken three minutes later and were completely disappearance only for bigeminy (Figure 13B). The patient pulse during the first ECG showed irregular regularity with regular dropped beats while pulse examination was irregular regularity during the second ECG. Bigeminy disappear without any medical interventions. The patient 
admitted in ICU as suspected acute pulmonary embolism. No recurrence of bigeminy on later serial ECG tracings. The patient was managed with standard therapy of pulmonary embolism. Electrolytes profile, thyroid function tests, full blood picture, kidney function tests, d-dimer, CXR, CTPA, and echocardiography were done. The patient left the hospital within one day of recovery.

\section{Case 14: Regular initial lead Bigeminy with AF}

A 50-year-old married Egyptian female housewife presented to the emergency room with palpitations. There was a history of chronic AF. The patient denied any history of cigarette smoking, drugs, any special diet/habit and any history of thyroid, or other relevant diseases, psychiatric problems, and exhaustion. Upon examination, the patient was conscious, alert with BP of $100 / 60 \mathrm{mmHg}$, the pulse rate of $120 \mathrm{bpm}, \mathrm{RR}$ of $17 \mathrm{bpm}$, the temperature of $37.1^{\circ} \mathrm{C}$, and $\mathrm{O}_{2}$ saturation with pulse oxymetry $94 \%$. No more relevant clinical data were noted during the clinical examination. The ECG recordings were showing bigeminy through most ECG leads (Figure 14A). ECG recordings were taken two minutes later and were completely normal (Figure 14B). The patient pulse during the first ECG showed irregular regularity with regular dropped beat while pulse examination was irregular regularity during the second ECG. Bigeminy only disappears without any medical interventions. The patient admitted in ICU for AF. No recurrence for bigeminy on later serial ECG tracings. Electrolytes profile, thyroid function tests, full blood picture, kidney function tests, and echocardiography were done. All results of the required investigations were normal. Echocardiography was left ventricular systolic dysfunction. The patient left the hospital after AF controlling.

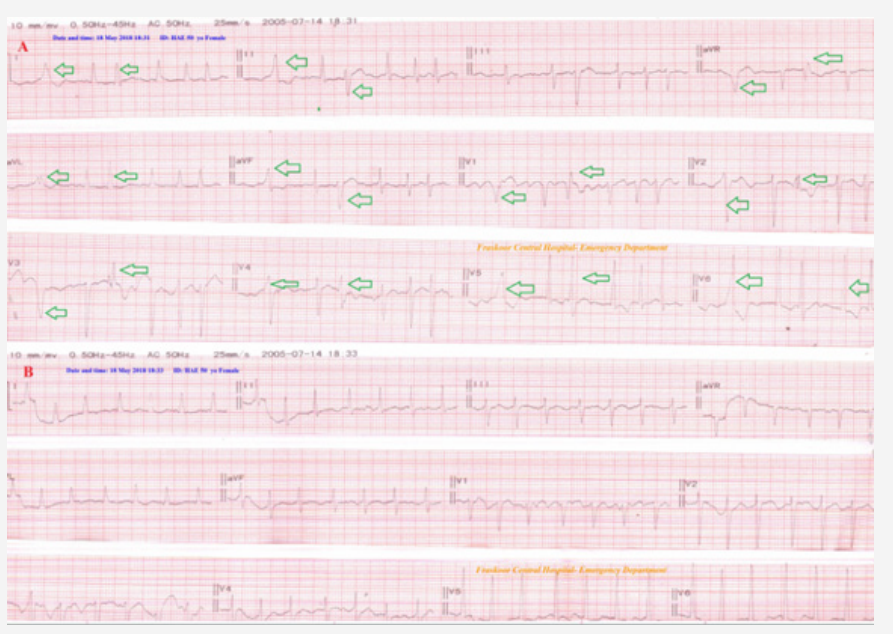

Figure 14 : A. The initial ECG tracing showing bigeminy through most ECG leads (green arrows indicate premature ventricular contraction (bigeminy) followed by AF.

B. The second ECG tracing (two minutes after the first ECG) showing only complete disappearance for bigeminy.

\section{Case 15: Sinus arrhythmia}

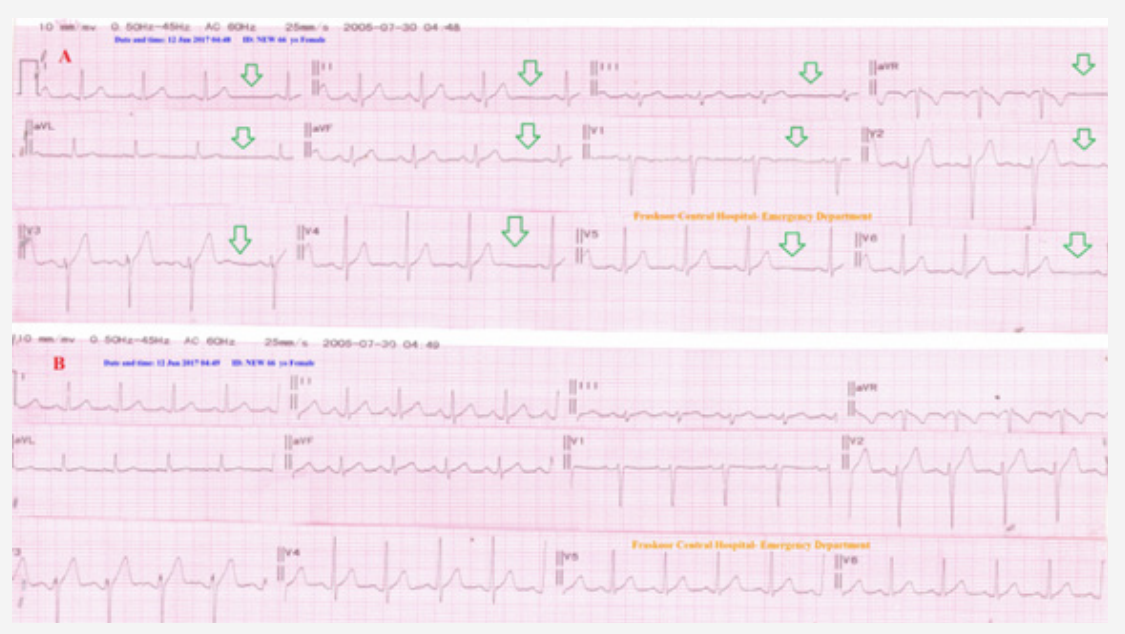

Figure 15 : A. the initial ECG tracing showing sinus arrhythmia (green arrows) and with VR; 92 bpm.

B. ECG tracing one minute later showing normalization of all above changes. 
A 66-year-old married housewife Egyptian female patient presented to the emergency room for follow up. Upon examination BP of 100/80 mmHg, the pulse of $90 \mathrm{bpm}$, RR of $15 \mathrm{bpm}$, the temperature of $36.8{ }^{\circ} \mathrm{C}$, and $\mathrm{O}_{2}$ saturation with pulse oxymetry $98 \%$. The ECG recordings were showing sinus arrhythmia (Figure 15A). ECG recordings were taken one minute later and were completely normal without any medications (Figure 15B). Electrolytes profile, thyroid function tests, full blood picture, and kidney function tests were done. All results of the required investigations were normal. Echocardiography was normal. The patient was only managed with reassurance. No recurrence for above ECG abnormalities on later serial ECG tracings follows up.

\section{Case 16: Sinus arrhythmia}

A 57-year-old married housewife Egyptian female patient presented to the emergency room for inguinal hernia preoperative preparation. Upon examination BP of $120 / 70 \mathrm{mmHg}$, the pulse of $76 \mathrm{bpm}$, RR of $16 \mathrm{bpm}$, the temperature of $36.4{ }^{\circ} \mathrm{C}$, and $\mathrm{O}_{2}$ saturation with pulse oxymetry $99 \%$. The ECG recordings were showing sinus arrhythmia (Figure 16A). ECG recordings were taken one minute later and were completely normal without any medications (Figure 16B). Electrolytes profile, thyroid function tests, full blood picture, and kidney function tests were done. All results of the required investigations were normal. Echocardiography was normal. The patient was only managed with reassurance. No recurrence for above ECG abnormalities on later serial ECG tracings follows up.

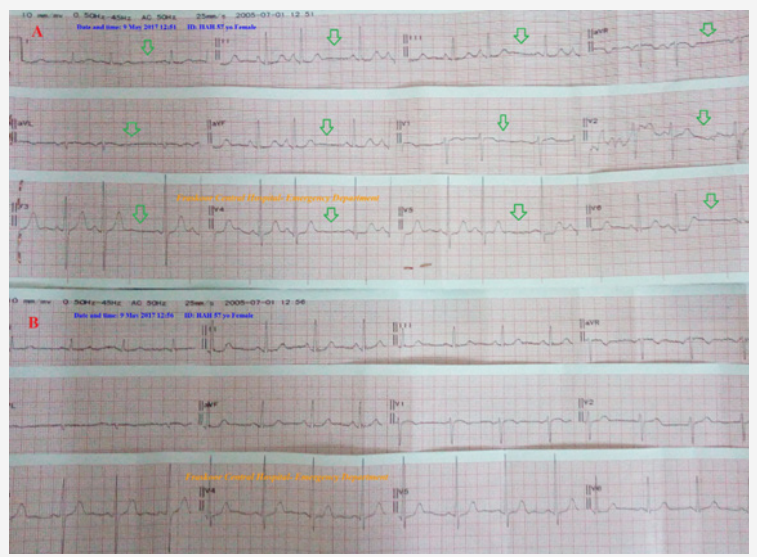

Figure 16 : A. the initial ECG tracing showing sinus arrhythmia (green arrows) with VR; 76 bpm.

B. ECG tracing one minute later showing normalization of all above changes.

\section{Case 17: Sinus arrhythmia}

A 34-year-old married worker Egyptian male patient presented to the emergency room for blood pressure follow up. Upon examination BP of 110/70 mmHg, the pulse of $66 \mathrm{bpm}$, RR of $14 \mathrm{bpm}$, the temperature of $36.8{ }^{\circ} \mathrm{C}$, and $\mathrm{O}_{2}$ saturation with pulse oxymetry 97\%. The ECG recordings were showing sinus arrhythmia (Figure 17A). ECG recordings were taken four minute later and were com- pletely normal without any medications (Figure 17B). Electrolytes profile, thyroid function tests, full blood picture, and kidney function tests were done. All results of the required investigations were normal. Echocardiography was normal. The patient was only managed with reassurance. No recurrence for above ECG abnormalities on later serial ECG tracings follows up.

Figure 17 : A. the initial ECG tracing showing sinus arrhythmia (green arrows) with VR; $66 \mathrm{bpm}$.

B. ECG tracing four minutes later showing normalization of all above changes. 


\section{Case 18: Sinus arrhythmia with anxiety}

A 85-year-old married housewife Egyptian female patient presented to the emergency room with palpitation. The patient had a recent history of anxiety. Upon examination; BP of 140/80 $\mathrm{mmHg}$, the pulse of $60 \mathrm{bpm}$, RR of $13 \mathrm{bpm}$, the temperature of $37.2^{\circ} \mathrm{C}$, and $\mathrm{O}_{2}$ saturation with pulse oxymetry $95 \%$. The ECG recordings were showing sinus arrhythmia (Figure 18A). ECG recordings were taken three minutes later and were completely normal without any medications (Figure 18B). Electrolytes profile, thyroid function tests, full blood picture, and kidney function tests were done. All results of the required investigations were normal. Echocardiography was normal. The patient was only managed with reassurance. No recurrence for above ECG abnormalities on later serial ECG tracings follows up.

Figure 18 : A. the initial ECG tracing showing sinus arrhythmia (green arrows) in all ECG leads except V6 due to movement artifact (blue arrows) with VR; $60 \mathrm{bpm}$.

B. ECG tracing three minutes later showing normalization of all above changes.

\section{Discussion and Results}

Age

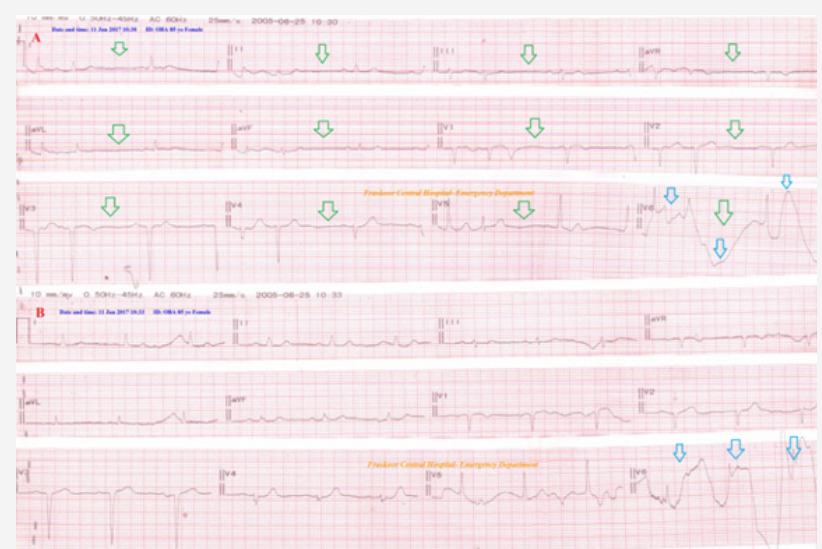

Figure 19 : Showing age averages.

Averages (Range;17-85, minimum;17, maximum; 85, mean; 54.83, median; 56, and mode; 56) (Figure 19)

\section{Sex and percentages (\%)}

$50 \%$ of the study cases are male ( 9 cases) and $50 \%$ are female (9 cases) (Figure 20)

\section{Occupation and percentages (\%)}
a. Student; $5.56 \%$ ( 1 case)
b. Teacher; $11.11 \%$ ( 2 cases $)$
c. Housewife; $50 \%$ ( 9 cases)

d. Farmer; $5.56 \%$ (1 cases)

e. Worker; $16.67 \%$ (3 cases)

f. Driver; $5.56 \%$ (1 case)

g. Carpenter; $5.56 \%$ ( 1 case) (Figure 21$)$

\section{The main complaint}
a. $\quad$ Chest pain; $22.22 \%$ (4 cases)
b. Palpitations; $16.67 \%$ (3 cases)
c. Dyspnea; $5.56 \%$ (1 case) 


\section{Sex and percentages [\%]}

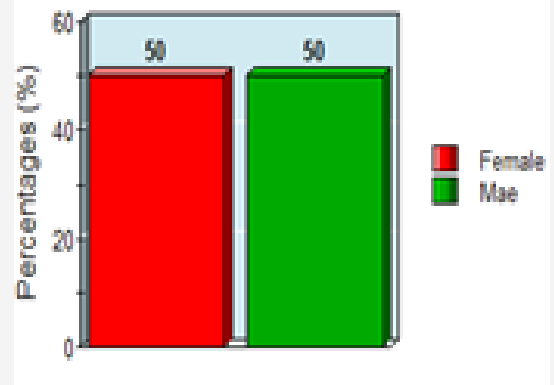

Ser

Figure 20 : Showing sex and percentages (\%).

\section{Various occupations percentage in the study}
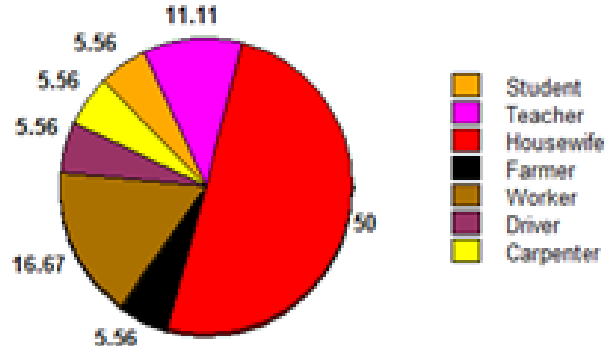

Figure 21 : Showing various occupations percentage in the study.
d. Headache; $5.56 \%$ (1 case)
f. On preoperative preparation; $5.56 \%$ (1 case)
e. Abdominal pain; $16.67 \%$ (3 cases)
g. On follow up; $27.78 \%$ (5 cases) (Figure 22)

\section{Percentages of main complaints in the study}

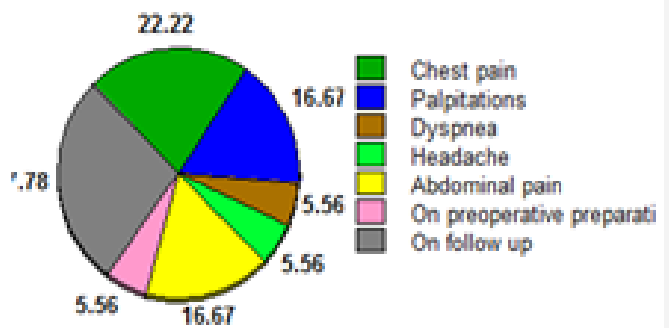

Figure 22 : Showing percentages of the main complaint in the study.

\section{The averages time elapsed between both ECG tracings} (minutes)

Averages (Range;1-4, minimum;1, maximum; 4, mean; 1.638, case) median; 1.25 , and mode; 1 )

\section{Basic diagnosis}
a. Cold; $11.11 \%$ ( 2 cases $)$
b. Cervical disc prolapses; $5.56 \% \%$ (1 case)
c. Myositis; $5.56 \% \%$ (1 case)

d. Anxiety; $16.67 \%$ (3 cases)

e. Cerebrovascular accident with hypertension; 5.56\% \% (1

f. Old myocardial infarction; $5.56 \% \%$ (1 case)

g. Renal pain; $5.56 \% \%$ (1 case)

h. Ischemic colitis; $5.56 \% \%$ (1 case)

i. Hypertension; $5.56 \% \%$ (1 case) 
j. Atrial fibrillation with suspected acute pulmonary embolism; $5.56 \% \%$ (1 case)

k. Atrial fibrillation; $5.56 \% \%$ (1 case) l. On follow up; $16.67 \% \%$ (3 cases)

m. On preoperative preparation; $5.56 \% \%$ (1 case) (Figure 23)

\section{Percentages of hasic diagnosis in the study}

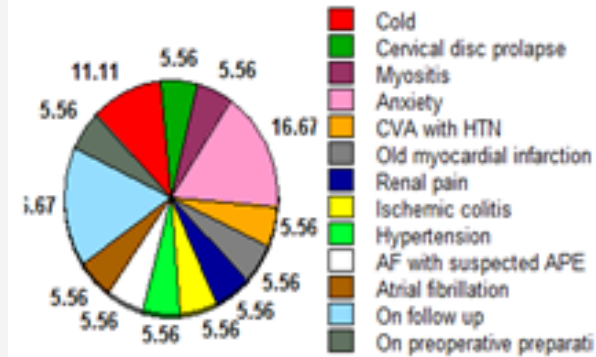

Figure 23 : Showing percentages of basic diagnosis in the study.

\section{Cardiac diagnosis}

a. Accelerated junctional rhythm; $22.22 \%$ (4 cases)

b. Mimic ST-segment elevation myocardial infarction with accelerated junctional rhythm; 5.56\% \% (1 case)

c. Sinus arhythmia; $22.22 \%$ (4 cases) d. Bigeminy; $11.11 \%$ ( 2 cases)

e. Trigeminy; $11.11 \%$ ( 2 cases $)$

f. Quaderigeminy; $16.67 \% \%$ (3 cases)

g. Pentageminy; $11.11 \%$ (2 cases) (Figure 24$)$

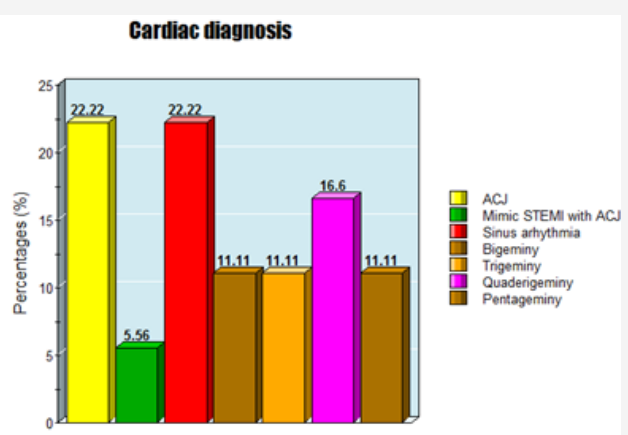

Figure 24 : Showing different cardiac diagnosis.

\section{Reassurance but with organic cause}

There is reassurance for all cases of the study (100\%). But the reassurance with presence of organic cardiac cause was only in seven cases of this study (38.89\%).

\section{The Characteristic Criteria for the "Passing phenomenon" are}

a. The new "Passing phenomenon" is a transient electrocardiographic change that spontaneously reversed within a few seconds to a few minutes without any medical interventions and apparent hemodynamic impact.

b. The eighteen reported cases were variable in diagnosis.

c. The cases cannot be included in one specific category. d. Not all cases have an organic cause.

e. The common denominator among the cases was the appearance of transient significant an electrocardiographic change with prompt and spontaneous disappearance of these changes within a few seconds to a few minutes without any medical interventions.

f. Otherwise, the medications for the basic pathology, all patients were lucky when no given medications or interference had happened.

g. All these ECG changes maybe not related to the diagnosis of relevant diseases.

h. These changes were transient. 
i. The included changes were completely reversed without any medical or procedural intervention.

j. The reversibility had happened within a few seconds to a few minutes.

k. Myocardial hyperexcitability and electrolytes change inside the maybe suggested theory for this phenomenon.

l. Don't neglect the significance of the serial ECG tracing copies.

m. Depending on the new phenomenon, some arrhythmias and anti-arrhythmic should be reviewed again.

n. Don't hurry to manage the ECG arrhythmic abnormalities unless be sustained.

o. Regards to ST-segment elevation, the physician should not urge to manage as ST-segment elevation or coronary artery vasospasm until established and confirmed the abnormalities.

p. Reassurance is immediate therapy.

q. The electrophysiological study is the future advised investigation.

\section{Conclusion and Recommendations}

a. The new "Passing phenomenon" is a transient electrocardiographic change that spontaneously reversed within a few seconds to a few minutes without any medical interventions and apparent hemodynamic impact.

b. Reassurance is the main immediate therapy unless there are otherwise sustaining electrocardiographic pathology.

c. Cellular electrolytes study may be advised future options for like these cases.

d. The electrophysiological study is the future advised investigation.

\section{Acknowledgement}

The author wishes to thank the critical care unit nurses who made extra ECG copies for helping.

\section{Conflict of Interest}

No conflict of interest.

\section{References}

1. Hafeez Y, Grossman SA (2019) Junctional rhythm. Stat Pearls Publishing LLC.

2. Semelka M, Gera J, Usman S (2013) Sick sinus syndrome: a review. Am Fam Physician 87(10): 691-696.

3. Trappe HJ (2010) Tachyarrhythmias, bradyarrhythmias and acute coronary syndromes. J Emerg Trauma Shock 3(2): 137-142.

4. Romhilt DW, Doyle M, Sagar KB, Hastillo A, Wolfgang TC, et al. (1982) Prevalence and significance of arrhythmias in long-term survivors of cardiac transplantation. Circulation 66(2 Pt 2):1219-1222.

5. Rodriguez RD, Schocken DD (1990) Update on sick sinus syndrome, a cardiac disorder of aging. Geriatrics 45(1): 26-30, 33-36.
6. Spodick DH (1992) Normal sinus heart rate: sinus tachycardia and sinus bradycardia redefined. Am Heart J 124(4): 1119-1121.

7. Thery C, Gosselin B, Lekieffre J, Warembourg H (1977) Pathology of sinoatrial node. Correlations with electrocardiographic findings in 111 patients. Am Heart J 93(6): 735-740.

8. Dobrzynski H, Anderson RH, Atkinson A, Borbas Z, D Souza A, et al. (2013) Structure, function and clinical relevance of the cardiac conduction system, including the atrioventricular ring and outflow tract tissues. Pharmacol 139(2): 260-288.

9. HauptmanPJ, Kelly RA (1999) Digitalis. Circulation 99(9): 1265-1270.

10. Kelly RA, Smith TW (1992) Recognition and management of digitalis toxicity. Am J Cardiol 69(18): 108G-118G.

11. Kishimoto N, Kinoshita I, Momota Y (2017) Junctional Rhythm Preoperatively and During General Anesthesia for Oral and Maxillofacial Surgery. Anesth Prog 64(3): 165-167.

12. Hillel Z, Landesberg G (2010) Electrocardiography. In: Miller RD (edr.), Miller's Anesthesia ( $7^{\text {th }}$ edn), Churchill Livingstone, USA, 1357-1386.

13. Antman EM, Wenger TL, Butler VP, Haber E, Smith TW (1990) Treatment of 150 cases of life-threatening digitalis intoxication with digoxinspecific Fab antibody fragments. Final report of a multicenter study. Circulation 81(6): 1744-1752.

14. Tuohy S, Saliba W, Pai M, Tchou P (2018) Catheter ablation as a treatment of atrioventricular block. Heart Rhythm 15(1): 90-96.

15. Kim D, Shinohara T, Joung B, Maruyama M, Choi EK, et al. (2010) Calcium dynamics and the mechanisms of atrioventricular junctional rhythm. J Am Coll Cardiol 56(10): 805-812.

16. Johnson Francis (2015) Low atrial rhythm-ECG.

17. Smith S (2017) An interesting finding, and absence of another, in an intoxicated patient found down.

18. Asakura Y, Mizuno T, Kandatsu N, Fujiwara Y, Akashi M (2009) Severe hypotension during thedevelopment of low-nodal junctional rhythm. ActaAnaesthe-siol Scand 53: 404-405.

19. Fujiwara Y, Asakura Y, Shibata Y, Nishiwaki K, Komatsu T (2006) A marked decrease in heart rate variability associated with junctional rhythm during anesthesia with sevoflurane and fentanyl. Acta Anaesthesiol Scand 50: 509- 511.

20. Cools E, Missant C (2014) Junctional ectopic tachycardia after congenital heart surgery. Acta Anaesthesiol Belg 65(1): 1-8.

21. Di Biase L, Gianni C, Bagliani G, Padeletti L (2017) Arrhythmias Involving the Atrioventricular Junction. Card Electrophysiol Clin 9(3): 435-452.

22. Silvestri NJ, Ismail H, Zimetbaum P, Raynor EM (2018) Cardiac involvement in the muscular dystrophies. Muscle Nerve 57(5): 707-715.

23. Sanders P, Kistler PM, Morton JB, Spence SJ, Kalman JM (2004) Remodeling of sinus node function in patients with congestive heart failure: reduction in sinus node reserve. Circulation 110(8): 897-903.

24. Dobrzynski H, Boyett MR, Anderson RH (2007) New insights into pacemaker activity: promoting understanding of sick sinus syndrome. Circulation 115(14): 1921-1932.

25. Arı H, Kahraman F, Bas HA, Arslan A (2015) Low atrial rhythm mimics myocardial infarction. Anatol J Cardiol 15(8): 675, 683.

26. Beinart SC (2017) Junctional Rhythm. Medscape.

27. Haruta D, Akahoshi M, Hida A, Sera N, Imaizumi M, et al. (2016) Prognostic Significance of Premature Ventricular. Ann Noninvasive Electrocardiol 21(2): 142-151.

28. Keany JE, Schraga ED (2013) Premature Ventricular Contraction Treatment \& Management.

29. Haist, Steven A Gomella, Leonard G (2004) Basic ECG Reading: Ventricular Arrhythmias, Clinician's pocket reference, Lange Clinical Science Series ( $10^{\text {th }}$ edn), McGraw-Hill, USA, pp. 390. 
30. Elsayed YMH (2019) Test the Termination of Regular Ischemic Premature Ventricular Contractions Variants with Nitroglycerin; A new Therapeutic and Diagnostic Test; Retrospective Observational Study (14-Report Cases). Emerg Med Trauma Care J.

31. Panico R, Powell W HA (1993) Guide to IUPAC Nomenclature of Organic Compounds. Blackwell Science, UK.

32. Elsayed YMH (2017) Test the antiarrhythmic effect of intravenous nitroglycerine on ischemic Premature Ventricular Contractions Bigeminy-Case report. The Egyptian Journal of Critical Care Medicine 5(3): 101-103.

33. Kerin N, Mori I, Levy MN (1975) Ventricular Quadrigeminy as a Manifestation of Concealed Bigeminy. Circulation 52: 1023-1029.

34. Umme Habiba Ferdaushi, Atahar Ali M, Shaila Nabi, Mainul Islam, Md Shamshul Alam, et al. (2016) Evaluation of Morphology of Premature Ventricular Contraction on 12-Lead Electrocardiogram. Bangladesh Heart Journal 31(2): 75-79.

35. Ng GA (2006) Treating patients with ventricular ectopic beats. Heart 92(11): 1707-1712.

36. Min Soo Ahn (2013) Current Concepts of Premature Ventricular Contractions. JLM 3(1): 26-33.

37. Kennedy HL, Whitlock JA, Sprague MK, Kennedy LJ, Buckingham TA, et al. (1985) Long-term follow-up of asymptomatic healthy subjects with frequent and complex ventricular ectopy. N Engl J Med 312(4): 193-197.

38. Messineo FC (1989) Ventricular ectopic activity: prevalence and risk. Am J Cardiol 64(20): 53J-56J.

39. Kostis JB, Mc Crone K, Moreyra AE, Gotzoyannis S, Aglitz NM, et al. (1981) Premature ventricular complexes in the absence of identifiable heart disease. Circulation 63(6): 1351-1356

40. Jin sheng Wang, Shen YG, Yin RP, Thapa S, Peng YP, et al. (2018) The safety of catheter ablation for premature ventricular contractions in patients without structural heart disease. BMC Cardiovasc Disord 18(1): 177.

41. Rakesh Latchamsetty, Frank Bogun (2016) Premature Ventricular Complex-induced Cardiomyopathy. Rev Esp Cardiol 69(4): 365-369.

42. Carlos Labadet (2015) Premature Ventricular Contractions strike Back. Rev Argent Cardiol 83: 552-554.

43. Elsayed YMH (2018) An electrocardiographic anxiety-induced quadrigeminy and re-assurance-Case report. The Egyptian Journal of Critical Care Medicine 6(1): 21-23.

44. Charles Jazra, Oussma Wazni, Wael Jaroudi (2015) Are premature ventricular contractions in patients without apparent structural heart disease really safe 2: 32-36.

45. Pedersen CT, Kay GN, Kalman J, Borggrefe M, Della Bella P, et al. (2014) EHRA/HRS/APHRS Expert Consensus on Ventricular Arrhythmias. Heart Rhythm 11(10): e166-e196.

46. Akdemir B, YarmohammadI H, Alraies MC, Adkisson WO (2016) Premature ventricular contractions: Reassure or refer? Cleve Clin J Med 83(7): 524-530
47. Yarlagadda RK, Iwai S, Stein KM, Markowitz SM, Shah BK, et al. (2005) Reversal of cardiomyopathy in patients with repetitive monomorphic ventricular ectopy originating from the right ventricular outflow tract. Circulation 112(8): 1092-1097.

48. Bogun F, Crawford T, Reich S, Koelling TM, Armstrong W, et al. (2007) Radiofrequency ablation of frequent, idiopathic premature ventricular complexes: comparison with a control group without intervention. Heart Rhythm 4(7): 863-867.

49. Sarrazin JF, Labounty T, Kuhne M, Crawford T, Armstrong WF, et al. (2009) Impact of radiofrequency ablation of frequent post-infarction premature ventricular complexes on left ventricular ejection fraction. Heart Rhythm. 6(11): 1543-1549.

50. Mountantonakis SE, Frankel DS, Gerstenfeld EP, Dixit S, Lin D, et al. (2011) Reversal of outflow tract ventricular premature depolarization induced cardiomyopathy with ablation: effect of residual arrhythmia burden and preexisting cardiomyopathy on outcome. Heart Rhythm 8(10): 1608-1614.

51. William H Sauer (2018) Normal sinus rhythm and sinus arrhythmia

52. Solos MP, Mc Comb D (2019) Sinus arrhythmia. Stat Pearls Publishing, USA.

53. Issa ZF, Miller JM, Zipes DP (2019) 8 - Sinus Node Dysfunction. Clinical Arrhythmology and Electrophysiology, A Companion to Braunwald's Heart Disease ( $3^{\text {rd }}$ edn), pp. 238-254.

54. Ed Burns (2019) Sinus arrhythmia.

55.Zebulon ZS, Meliones C, Idriss SF (2019) 27 - Arrhythmias and Pacing. Critical Heart Disease in Infants and Children ( $3^{\text {rd }}$ edn), Elsevier Inc.

56. Shindler DM, Kostis JB (2009) Chapter 13 - Electrocardiographic Technology of Cardiac Arrhythmias. Basic Science, Technical Considerations, and Clinical Aspects ( $3^{\text {rd }}$ edn), Sleep Disorders Medicine, pp. 182-187.

57. Kaushal P, Taylor JA (2002) Inter-relations among declines in arterial distensibility, baroreflex function and respiratory sinus arrhythmia. J Am Coll Cardiol 39(9): 1524-1530.

58. Coker R, Koziell A, Oliver C, Smith SE (1984) Does the sympathetic nervous system influence sinus arrhythmia in man? Evidence from combined autonomic blockade. J Physiol 356: 459-464.

59. Piepoli M, Sleight P, Leuzzi S, Valle F, Spadacini G, et al. (1997) Origin of respiratory sinus arrhythmia in conscious humans. An important role for arterial carotid baroreceptors. Circulation 95(7): 1813-1821.

60. Smith SA (1982) Reduced sinus arrhythmia in diabetic autonomic neuropathy: diagnostic value of an age-related normal range. Br Med J 285(6355): 1599-1601.

61. Barbosa Filho J, Barbosa PR, Cordovil I (2002) Nonrespiratory sinus arrhythmia. Arq Bras Cardiol 78(4): 406-411

62. Deboor SS, Pelter MM, Adams MG (2005) Nonrespiratory sinus arrhythmia. Am J Crit Care 14(2): 161-162. 